OPEN ACCESS

Edited by:

Taruna Madan,

National Institute for

Research in Reproductive

Health (ICMR), India

Reviewed by:

Anthony George Tsolaki,

Brunel University London,

United Kingdom

Kenneth Reid,

University of Oxford,

United Kingdom

*Correspondence:

Jiu-Yao Wang

a122@mail.ncku.edu.tw;

Lawrence S. H. Wu

Ishwu@hotmail.com

Specialty section:

This article was submitted to

Molecular Innate Immunity,

a section of the journal

Frontiers in Immunology

Received: 28 February 2018

Accepted: 21 June 2018

Published: 02 July 2018

Citation:

Hsieh M-H, Ou C-Y, Hsieh W-Y,

Kao H-F, Lee S-W, Wang J-Y

and Wu LSH (2018) Functional

Analysis of Genetic Variations

in Surfactant Protein D in

Mycobacterial Infection and Their Association With Tuberculosis.

Front. Immunol. 9:1543.

doi: 10.3389/fimmu.2018.01543

\section{Functional Analysis of Genetic Variations in Surfactant Protein D in Mycobacterial Infection and Their Association With Tuberculosis}

\author{
Miao-Hsi Hsieh", Chih-Ying Ou', Wen-Yu Hsieh ${ }^{3}$, Hui-Fang Kao ${ }^{4}$, Shih-Wei Lee ${ }^{5}$, \\ Jiu-Yao Wang ${ }^{1,3,6 *}$ and Lawrence S. H. Wu $u^{4,7 *}$
}

${ }^{1}$ Institute of Basic Medical Science, College of Medicine, National Cheng Kung University, Tainan, Taiwan, ${ }^{2}$ Institute of Clinical Medicine, College of Medicine, National Cheng Kung University, Tainan, Taiwan, ${ }^{3}$ Institute of Biochemistry and Molecular Biology, College of Medicine, National Cheng Kung University, Tainan, Taiwan, ${ }^{4}$ Allergy and Clinical Immunology Research (ACIR) Center, College of Medicine, National Cheng Kung University, Tainan, Taiwan, ${ }^{5}$ Chest Medicine, General Taoyuan Hospital, Taoyuan, Taiwan, ${ }^{6}$ Institute of Respiratory Research, The First Affiliated Hospital of Guangzhou Medical University, Guangzhou, China, ' Graduate Institute of Biomedical Sciences, China Medical University, Taichung, Taiwan

Surfactant proteins (SPs)-A and -D are C-type lectins of the collectin family and function in the clearance of infectious particles in the lungs. Some polymorphisms of SPs that give rise to amino acid changes have been found to affect their function. Several SP-A gene polymorphisms have been reported to be associated with respiratory infection diseases, such as tuberculosis (TB). However, the relationship between surfactant proteins D (SP-D) polymorphisms and TB is still unclear. To study the associations between SP-D polymorphisms and TB, the correlations of SP-D polymorphisms with TB were examined in a case-control study, which included 364 patients with TB and 177 control subjects. In addition, we cloned two major SP-D exonic polymorphism C92T (rs721917) and A538G (rs2243639) constructs and used these for in vitro assays. The effects of SP-D polymorphisms on agglutination and other interactions with Mycobacterium bovis bacillus Calmette-Guérin ( $M$. bovis BCG) were evaluated. In comparison with SP-D 92C (amino acid residue 16, Threonine), our results showed that SP-D 92T (amino acid residue 16 , Methionine) had a lower binding ability to $M$. bovis BCG, a lower capacity to inhibit phagocytosis, lesser aggregation, poorer survival of bacillus Calmette-Guérin (BCG)-infected $\mathrm{MH}-\mathrm{S}$ cells, and less inhibition of intracellular growth of $M$. bovis BCG. The case-control association study showed that the 92T homozygous genotype was a risk factor for TB. However, a lesser effect was seen for polymorphism A538G. In conclusion, the results of functional and genetic analyses of SP-D variants consistently showed that the SP-D 92T variant increased susceptibility to TB, which further confirmed the role of SP-D in pulmonary innate immunity against mycobacterial infection.

\footnotetext{
Keywords: infection, Mycobacterium, single-nucleotide polymorphisms, surfactant protein $\mathrm{D}$, tuberculosis
}

Abbreviations: CRD, carbohydrate recognition domain; HMW, high molecular weight; LMW, low molecular weight; LAM, lipoarabinomannan; MTB, Mycobacterium tuberculosis; MOI, multiplicity of infection; rSP-D, recombinant SP-D; SNPs, single-nucleotide polymorphisms; SP-D, surfactant proteins D; TB, tuberculosis; M. bovis BCG, Mycobacterium bovis bacillus Calmette-Guérin. 


\section{INTRODUCTION}

Pulmonary tuberculosis (TB), caused by Mycobacterium tuberculosis (MTB), is a global public health issue, as it is the leading cause of death among infectious diseases in many countries. Though TB has diminished over a long period of time, it has reappeared and has become a serious concern once again in many regions. The resurgence of $\mathrm{TB}$ is also accompanied by increasing numbers of cases of multidrug-resistant $\mathrm{TB}$ due to the development of extensively drug-resistant bacterial strains $(1,2)$. A study estimated that more than $30 \%$ of the world's population has been infected with $M T B$, and the infection kills approximately two million people annually (3). In Taiwan, there were more than 12,000 cases (55/100,000 population) of TB in 2011 alone, and at least 600 deaths (2.8/100,000 population) were caused by TB (4). The development of and susceptibility to TB involves complicated host environment-pathogen interactions, and genetic components have been suggested to be involved in the process. Recently, more evidence has been uncovered to indicate that individuals with certain genetic chrematistics are particularly vulnerable to TB. In addition, data accumulated from different types of studies, such as twin studies $(5,6)$, genome-wide linkage analyses $(7-10)$, and genome-wide association studies (11-13), have demonstrated that individuals with particular genetic variations are at high risk of TB (14). In fact, among people infected with TB, only $10 \%$ develop active pulmonary TB, implying that polymorphisms of genes associated with host immune responses may be key to the development of pulmonary TB (15).

C-type lectins, collagen-like calcium-dependent pulmonary collectins, are some of the molecules correlated with innate host immune responses. These include lung surfactant proteins (SP)$\mathrm{A}$ and $-\mathrm{D}$ and pattern recognition molecule-mannose-binding lectin/protein (MBL or MBP) (16). These molecules play important roles in the host cellular response against mycobacterial infections (17). During the course of MTB infection, SP-A mediates the adherence of MTB to human alveolar macrophages and induces mannose receptor activity, which further increases their phagocytic uptake of MTB (18). Surfactant proteins D (SP-D), however, directly interacts with $M T B$, resulting in reduction of MTB phagocytosis (19), and the inhibition effect on MTB uptake by macrophages is not associated with the bacterial agglutination process (20). Incubation of MTB with SP-D results in reduced uptake of the bacteria by macrophages (19) and limits $M T B$ growth inside cells by facilitating the fusion of MTB phagosomes with lysosomes of cells (21). Allelic variations of SP-A and SP-D genes have been reported to be target candidates in several infectious diseases, including TB, as they are directly involved in the process of lung pathogen clearance (22). A study of patients of a Mexican population showed that several SP flanking marker alleles are associated with an increased risk of susceptibility to TB (23). Intronic single-nucleotide polymorphisms (SNPs) in the collagen region of SP-A2 and intragenic SNPs in SP-A1 and SP-A2 have also been demonstrated to contribute to the risk of susceptibility to $\operatorname{TB}(24,25)$. Thus, based on the aforementioned research and other previous studies, it has been proposed that surfactant polymorphisms may alter splicing regulation and impact on mRNA maturation (25). However, many of the underlying mechanisms are still unclear. Recently, Yang et al. (26) reported that SNPs in SP-A, particularly at alleles of amino acids $91(\mathrm{G})$ and $140(\mathrm{~T})$, are associated with $\mathrm{TB}$ in the Han population in China. Studies of protein constitution in bronchoalveolar lavage and surfactant gene polymorphisms have suggested that SP-D is directly linked to several pulmonary inflammatory diseases $(16,27)$. SP-D polymorphisms have also been found to be connected to severe respiratory syncytial virus infection, with the rs721917 SP-D allele coding for methionine (28). Our previous study also showed that genetic variants of pulmonary SP-D could determine serum levels of SP-D and predict the outcome of chronic obstructive pulmonary disease in a Chinese population (29). To the best of our knowledge, only one study of a Mexican population has identified an association between SP-D polymorphisms and susceptibility to TB (23). However, this research only investigated selective loci, without whole SP-D gene analysis, and lacked functional study of the correlations between SP-D polymorphisms and MTB infection. In fact, little comprehensive work has been carried out to analyze the relationships between genetic variants of SP-D proteins and host immunity against $M T B$ infection.

In this study, the effects of functional (in exon, non-synonymous) polymorphisms of SP-D on the interaction between SP-D and Mycobacterium bovis bacillus Calmette-Guérin (M. bovis BCG) were investigated. Furthermore, the associations between SP-D functional polymorphisms and susceptibility to TB were also investigated. This work provides evidence that utilizing functional DNA variants of SP-D in clinical association studies, instead of markers of SNPs, advances our understanding of surfactant genetic susceptibility and innate defense mechanisms to pulmonary TB and other lung diseases.

\section{MATERIALS AND METHODS}

\section{Clinical Sample Collection}

A total of 364 patients who were treated for active TB at the General Taoyuan Hospital (Taoyuan, Taiwan) and National Cheng Kung University Hospital (Tainan, Taiwan) between 2010 and 2012 were surveyed consecutively. The inclusion criteria were: adult patients newly diagnosed with active $\mathrm{TB}$, evident lesions of TB on simple X-ray and computed tomography images, and positive results of sputum smears and cultures for mycobacteria. Patients with HIV infection were excluded from this study. The control group comprised 177 volunteer subjects without active TB or a history of TB. Written informed consent was obtained from each patient and volunteer enrolled in this study. The study protocol conformed to the ethical guidelines of the 1975 Declaration of Helsinki and was approved by the Ethics Committees of Taoyuan General Hospital and National Cheng Kung University Hospital.

\section{DNA Extraction and SNP Genotyping}

Genomic DNA was extracted from oral swabs collected from the enrolled TB patients and non-TB subjects using a QIAamp DNA Mini Kit (QIAGEN, Valencia, CA, USA) according to the manufacturer's instructions. The extracted genomic DNA was analyzed 
using agarose gel electrophoresis, quantitatively determined by spectrophotometry, and stored at $-80^{\circ} \mathrm{C}$ until use. Genotyping was performed on the two major non-synonymous SNPs of SP-D, rs721917 (C92T) and rs2243639 (A538G), for genetic association analysis. The SNPs were genotyped using the high-throughput, 384-microtiter plate MassARRAY System, SEQUENOM, according to the manufacturer's protocol. In brief, DNA containing the SNP site of interest was amplified, followed by performance of the homogenous MassEXTEND assay, in which label-free primer extension chemistry was used to generate allele-specific diagnostic products. The allele-specific diagnostic products have a unique molecular weight, and this can be distinguished through the application of matrix-assisted laser desorption ionization time-of-flight mass spectrometry.

\section{Chemicals and Plasmids}

QIAprep Miniprep and QIAGEN plasmid Midi Kit for plasmid preparation were obtained from QIAGEN. Restriction enzymes including Hind III and Sac II were obtained from TaKaRa Bio. Dpn I for site mutagenesis was obtained from New England Biolabs. The vector pcDNA3.1/myc-His B was purchased from Invitrogen.

\section{Site-Directed Mutagenesis of Human SP-D}

The cDNA fragment encoding human SP-D was amplified by PCR using primers (forward: 5'-CCCAAGCTTGCCATGCTG CTCTTCCTCCTCTCTG-3' containing a $5^{\prime}$ HindIII adaptor; reverse: 5' -GCTCTAGATCAGAACTCGCAGACCACAAG-3' containing a 5' SacII adaptor) and cloned into HindIII and SacII sites, leading to the formation of recombinant plasmid pcDNA3.1/ myc-His B-SP-D (92T/538G). Site-directed mutagenesis was performed using mutagenic primers of SP-D 92T (forward: 5'-GA CCTACTCCCACAGAACAACGCCCAGTGCTTG-3'; reverse: 5' -CAAGCACTGGGCGTTGTTCTGTGGGAGTAGGTC-3' and SP-D538G forward: 5'-GTGGAGTCCCTGGAAACACAGG GGCAGC-3'; reverse: 5'-CAAGCACTGGGCGTTGTTCTGTG GGAGTAGGTC-3'). The recombinant plasmids were then transformed into Escherichia coli XL-1 Blue competent cells (Invitrogen) by the heat shock method, and grown in LB broth at $37^{\circ} \mathrm{C}$. Colonies of transformed cells were selected for further gel electrophoresis.

\section{Construction and Expression of Recombinant SP-D (rSP-D) Site-Directed Mutants}

The construction of recombinant plasmid pcDNA3.1/myc-His B-SP-D (92T/538G) is illustrated in Figure 1A. Site-directed mutagenesis of pcDNA3.1/myc-His B-SP-D (92T/538G) resulted in four variants (Figure 1B). SNPs in residues 92 and 538 resulted in changes in amino acids (Figure 1C). Gel electrophoresis of the four mutagenic DNA products after transformation with Hind II and Sac II diagnosis digestion is demonstrated in Figure 2A. The results of DNA sequencing of the four variants of SP-D are illustrated in Figure 2B. Expression and quantification of the four rSP-D site-directed mutants were confirmed by western blot (Figure 2C) and SP-D ELISA analysis (data not shown).
A

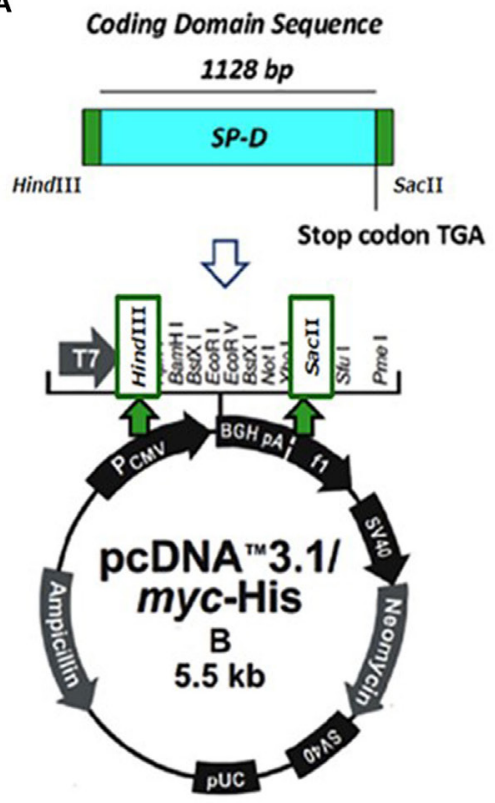

B

a. $p c D N A 3.1-S P-D(92 T / 538 A)$

b. pCDNA3.1-SP-D(92T/538G)

c. pCDNA3.1-SP-D(92C/538A)

d. PCDNA3.1-SP-D(92C/538G)

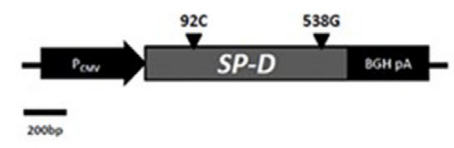

C

\begin{tabular}{lccc}
\hline Region & Domain & Allele & Amino acid \\
\hline Exon 1 & N-term & $92 \mathrm{~T} \rightarrow 92 \mathrm{C}$ & $11 \mathrm{Met} \rightarrow 11 \mathrm{Thr}$ \\
\hline Exon 4 & Collagen & $538 \mathrm{G} \rightarrow 538 \mathrm{~A}$ & $160 \mathrm{Ala} \rightarrow 160 \mathrm{Thr}$ \\
\hline
\end{tabular}

FIGURE 1 | (A) Illustration of the construction of recombinant full-length human surfactant proteins D (SP-D) plasmid into which the SP-D gene was inserted at the multiple cloning site of a pcDNA 3.1/myc-His B vector between Hind III and Sac II. (B) Four recombinant SP-D (rSP-D) plasmids that carried two major singlenucleotide polymorphisms under four different combinations (92T/538A, 92T/538G, 92C/538A, and 92C/538G) were created using gene cloning and site-directed mutagenesis. (C) Corresponding sites of nucleotides and amino acids in different polymorphisms of SP-D variants. 


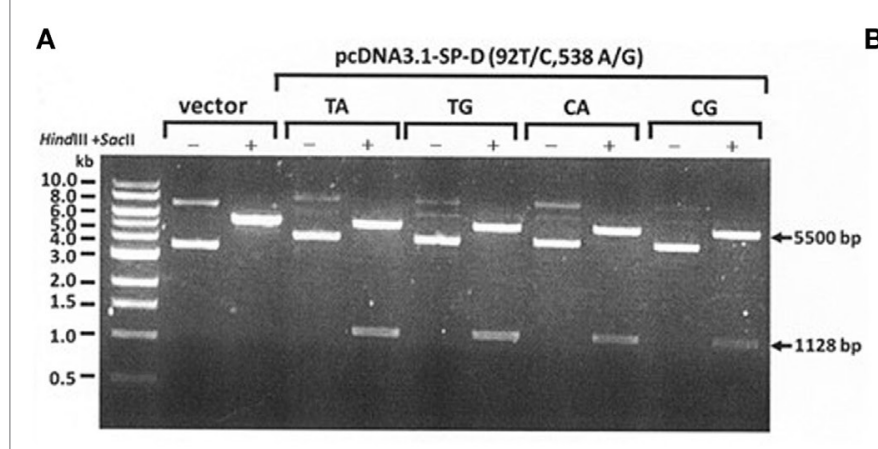

C

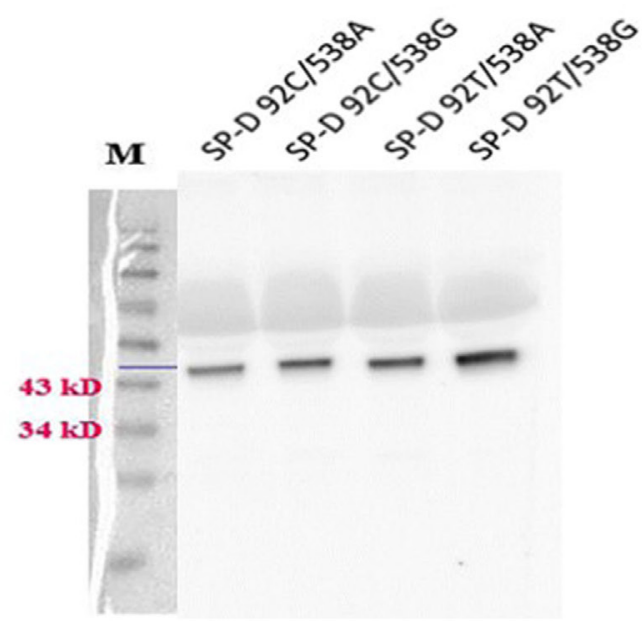

B

PCDNA3.1-SP-D(92C,538A)
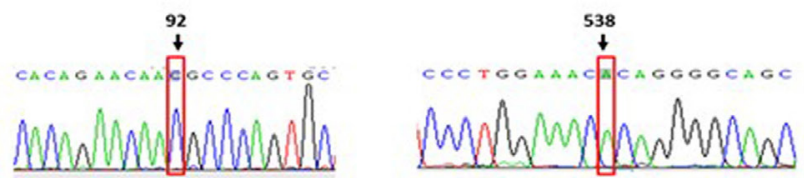

PCDNA3.1-SP-D(92C,538G)
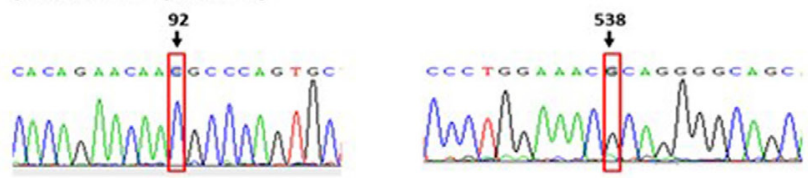

PCDNA3.1-SP-D(92T,538A)
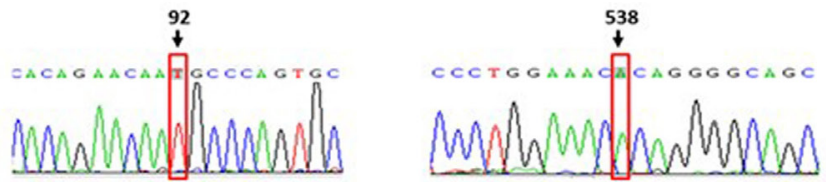

pcDNA3.1-SP-D(92T,538G)
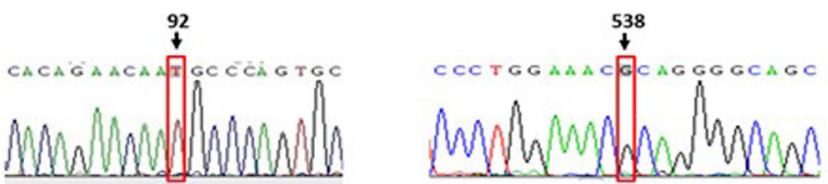

FIGURE 2 | (A) Double digestion of the vector and four surfactant proteins D (SP-D) gene variant plasmids using the restriction enzymes Hind III and Sac II for diagnostic digestion (TA: 92T/538A, TG:92T/538G, CA: 92C/538A, and CG: 92C/538G). (B) Sequencing chromatograms of the pcDNA3.1-SPD variant constructs confirmed the generation of four recombinant SP-D (rSP-D) genes using site-directed mutagenesis. (C) Western blots of the four rSP-D proteins from transfected HEK 293 cells showing the correct sizes of the proteins.

\section{Expression and Quantification of Four Variants of rSP-D}

Four variants of the rSP-D gene were transfected into human embryonic kidney 293 (HEK 293) cells (ATCC No. CRL-1573) using Lipofectamine LYX ${ }^{\mathrm{TM}}$ Reagent (Invitrogen). Culture medium (serum-free Dulbecco's Modified Eagle Medium) from transfected HEK 293 cells was collected for further western blot analysis. An SP-D ELISA kit (Hycult) was used for final quantification of the four rSP-D variants.

\section{Cell and Bacterial Culture}

The alveolar macrophage cell line derived from BALB/c mice (MH-S cells; American Type Culture Collection, Rockville, MD, USA) used in the study was a continuous cell line of murine alveolar macrophages established after transformation of cells obtained by bronchoalveolar lavage from BALB/c mice with simian virus 40 . The cells were cultured in RPMI 1640 medium (Gibco BRL, Gaithersburg, MD, USA) supplemented with $10 \%$ fetal bovine serum, $50 \mathrm{U} / \mathrm{ml}$ penicillin, $0.05 \mathrm{mg} / \mathrm{ml}$ streptomycin, $20 \mathrm{mM}$ L-glutamine, $10 \mathrm{mM}$ HEPES, $10 \mathrm{mM}$ sodium pyruvate, $1 \%$ glucose, and $50 \mu \mathrm{M} 2$-mercaptoethanol at $37^{\circ} \mathrm{C}$ in $5 \% \mathrm{CO}_{2} / 95 \%$ air.
M. bovis BCG was cultured on Middlebrook 7H11 agar (BD) at $37^{\circ} \mathrm{C}$ in an incubator. Bacteria were harvested, and pellets were suspended in PBS plus $10 \%$ glycerol. Aliquots $\left(\sim 5 \times 10^{8} /\right.$ vial $)$ were stored at $-80^{\circ} \mathrm{C}$. Before infection, the bacteria supplemented with glycerol were replaced with a final concentration of $0.05 \% \mathrm{v} / \mathrm{v}$ Tween 80 and $2 \mathrm{mM} \mathrm{CaCl}_{2} \mathrm{PBS}$ to reduce bacillus Calmette-Guérin (BCG) clumping and for subsequent preparation for SP-D binding. To avoid effects on the viability of $M$. bovis BCG, penicillin and streptomycin were not added to the culture medium of the MH-S cells (Sigma-Aldrich, St. Louis, MO, USA) that were prepared for infection.

\section{M. bovis BCG Infection}

A multiplicity of infection (MOI) of 1 (1 bacterium per MH-S cell) was used for all the experiments, with the exception of the phagocytosis assay, in which an MOI of 5 was used. Quantitative bacteria in different cell culture plates (6-well: $10^{5}, 12$-well: $5 \times 10^{4}$, 24-well: $2.5 \times 10^{4}$, and 96-well: $5 \times 10^{3}$ ) were supplemented with $100 \mathrm{mM}$ glucose or $100 \mathrm{mM}$ maltose, with or without $10 \mathrm{mM}$ EDTA, and with one of the four variants of SP-D $(0.5$ or $1 \mu \mathrm{g} / \mathrm{ml})$. One hour after infection, un-infected $\mathrm{MH}-\mathrm{S}$ cells were washed 
twice with PBS, and the same conditional medium was used for subsequent analysis.

\section{Solid-Phase Bacterial ELISA}

Lipoarabinomannan (LAM) from M. bovis BCG, which belongs to the class of LAMs capped with mannosyl residues, has a similar structure and immune-regulatory function to MTB (19). LAM also serves as a major binding molecule for SP-D (30). Based on this, we examined the roles of the four variants of rSP-D in the binding to $M$. bovis BCG. In experiments designed to determine the saturable binding activity of the four SP-D variants to $M$. bovis BCG, bacterial suspensions ( $10^{6}$ bacteria in $100 \mu \mathrm{TBS}$ ) were first dried at $37^{\circ} \mathrm{C}$ in an incubator for $7 \mathrm{~h}$, and then exposed to ultraviolet light for $2 \mathrm{~h}$ to kill any viable bacteria. After blocking with $3 \%$ BSA overnight at $4^{\circ} \mathrm{C}, 1$ and $10 \mu \mathrm{g} / \mathrm{ml}$ of the four variants of SP-D in PBS with $2 \mathrm{mM} \mathrm{CaCl}_{2}$ were added with or without $10 \mathrm{mM}$ EDTA, and with $100 \mathrm{mM}$ maltose or $100 \mathrm{mM}$ glucose. The results from 1 and $10 \mu \mathrm{g} / \mathrm{ml}$ of the four variants of SP-D were similar, then $1 \mu \mathrm{g} / \mathrm{ml}$ was used to further experiments. After incubation for $1 \mathrm{~h}$ at room temperature, the bacterial suspensions were washed and then incubated with a 1:5,000 dilution of antiSP-D antibody for $2 \mathrm{~h}$ at room temperature. After incubation, the suspensions were washed again and then incubated with a 1:3,000 dilution of goat anti-mouse IgG conjugated to HRP for $2 \mathrm{~h}$ at room temperature. Finally, TMB substrate was added after washing. Substrate development was stopped after $12 \mathrm{~min}$ by $2 \mathrm{~N}$ $\mathrm{H}_{2} \mathrm{SO}_{4}$, and the absorbance of individual wells was determined at O.D. $450 \mathrm{~nm}$ using a SpectraMax M2 ELISA reader (Molecular Devices, Sunnyvale, CA, USA).

\section{Phagocytosis of $M$. bovis BCG by MH-S Cells}

A Vybrant Phagocytosis Assay Kit (V6694; Invitrogen ${ }^{\mathrm{TM}}$, Thermal Fisher Scientific) was used to measure the phagocytosis of mycobacteria by $\mathrm{MH}-\mathrm{S}$ cells. Briefly, bacteria $\left(10^{9} / \mathrm{ml}\right)$ were labeled by incubation with FITC (Sigma-Aldrich) at $1 \mu \mathrm{g} / \mathrm{ml}$ in PBS for $2 \mathrm{~h}$ at $37^{\circ} \mathrm{C}$. Thereafter, FITC-labeled bacteria were washed twice with PBS, then opsonized, and used for infection as described above. Macrophages infected by FITC-labeled M. bovis BCG were analyzed and gated using flow cytometry to assess the effects of the four rSP-D variants on the phagocytosis of MH-S cells. Briefly, MH-S cells $\left(10^{5}\right)$ were incubated with FITC-labeled M. bovis BCG $\left(5 \times 10^{5} \mathrm{CFU}\right)$ in a 6 -well cell culture plate $(\mathrm{BD}$, Franklin Lakes, NJ, USA) in the absence or presence of the four SP-D variants at concentrations ranging from 0.5 to $1 \mu \mathrm{g} / \mathrm{ml}$. After incubation for $1 \mathrm{~h}, \mathrm{MH}-\mathrm{S}$ cells were scraped out after fixation with $4 \%$ formaldehyde PBS ( $\mathrm{pH} 7.2$ ) and examined using a flow cytometer (FACSCalibur; BD, San Jose, CA, USA) equipped with an argon laser that emits at $488 \mathrm{~nm}$. At least $1 \times 10^{4}$ of cells with FITC-labeled bacteria were counted in the FACS analysis.

\section{Agglutination and Cell Migration of $M$. bovis BCG-Infected MH-S Cells}

To further characterize the effects of the four SP-D variants on the agglutination of $\mathrm{MH}-\mathrm{S}$ cells after mycobacterial infection as described above, micrographs were taken 2 days after infection using an Olympus IX-71 inverted microscope. The area of the agglutinates was measured using ImageJ $1.39 \mathrm{~g}$ software (National Institutes of Health, Bethesda, MD, USA). The diameter was estimated from the area based on the circular form of the aggregates according to the formula Area $=\left(R \times D^{2}\right) / 4$, where $R=$ ratio of a circle's circumference to its diameter and $D=$ diameter. To determine the effects of the four variants of SP-D on macrophage cell migration, $2.5 \times 10^{4} \mathrm{MH}-\mathrm{S}$ cells were seeded in a 24-well cell culture plate and infected with $M$. bovis BCG as described above. The culture medium was harvested 2 days after infection and added into a new 24-well cell culture plate equipped with Millicell Hanging Cell Culture Inserts ( $3 \mu \mathrm{m}$ pore size, Millipore). Each insert was seeded with $5 \times 10^{4}$ MH-S cells. After 1 day of culture, the inserts were removed and observed using an Olympus IX-71 inverted microscope to count the cells migrated through the membrane. The chemoattractive $\mathrm{MH}-\mathrm{S}$ cells were quantified using ImageJ $1.39 \mathrm{~g}$ software.

\section{M. bovis BCG-Infected MH-S Cells Survival Assay}

Quantitative bacteria in a 96-well cell culture plate $\left(5 \times 10^{3}\right.$ $\mathrm{MH}-\mathrm{S} / 100 \mu \mathrm{l}$ medium) were infected with $M$. bovis BCG as described above. Two days after infection, surviving MH-S cells were evaluated using a Cell Counting Kit-8 (CCK-8) (Dojindo Laboratories, Kumamoto, Japan) with a SpectraMax M2 ELISA reader (Molecular Devices).

\section{M. bovis BCG Intracellular Growth Assay}

To determine the effects of the four SP-D variants on the intracellular viability of bacteria after phagocytosis by macrophages, $100 \mu \mathrm{l}$ RIPA buffer (10×, Millipore) were added to the culture plate 2 days after $\mathrm{MH}-\mathrm{S}$ cells had been infected $\left(5 \times 10^{4}\right.$ in a 12 -well cell culture plate). Aliquots of $\mathrm{MH}$-S cell lysate were then plated onto $7 \mathrm{H} 11$ agar (BD) to quantify the intracellular growth of viable $M$. bovis BCG. Colony-forming units were counted after 28 days of bacteria culture.

\section{Statistical Analysis}

Results are given as means \pm SEs. ANOVA was used to compare the rSP-Ds. All $P$ values less than 0.05 were considered significant. Statistical analysis was carried out using Prism, version 5 (GraphPad Software, San Diego, CA, USA). In the genetic association study, the quality of the genotype data was evaluated by Hardy-Weinberg equilibrium proportion tests. $\chi^{2}$ tests were used for association analyses. Odds ratios and 95\% confidence intervals were calculated from contingency tables. SNPs showing significant associations $(P$ value $\leq 0.05)$ in the tests were further evaluated using logistic regressions adjusted for age and gender in odds ratio analysis. Statistical analyses for the association study were performed using SPSS 17.0 software (SPSS Inc., Chicago, IL, USA).

\section{RESULTS}

\section{Subject Characterization}

Surfactant proteins D genetic polymorphisms are most often associated with pulmonary diseases, and our hypostatized genetic variants of SP-D may have different levels of contribution to the 
pathogenesis of TB. Therefore, we analyzed the genotype and allele frequencies of SP-D in TB- and non-TB-infected patients. 364 case subjects with diagnosed TB and 177 control subjects without a history of TB infection were enrolled. The mean age of the cases and controls was 55.22 and 57.75 years, respectively (Table 1). Cases were more likely to be male $(P<0.0001)$. There was no significant difference between the groups regarding age $(P>0.05)$.

\section{Variants of SP-D Associated With TB}

The genotype and allele frequencies of C92T (SNP ID: rs721917) and A538G (SNP ID: rs2243639) could not be derived from the Hardy-Weinberg principle. The association between TB and rs721917 genotypes was marginally significant $(P=0.050)$, and this significance was reduced after adjusting for age and gender (Table 2). However, odds ratio analysis showed that subjects with TT homozygous rs721917 alleles had an increased risk of susceptibility to TB ( $\mathrm{OR}=1.95, P=0.045$; Table 2 ), as the homozygous methionine amino acid at SP-D polypeptide position 11 was more frequent in TB patients than in non-TB subjects. The statistically significant association between rs2243639 and TB was increased when the results were adjusted for age and gender (from 0.061 to 0.033; Table 2). The homozygous AA (amino acid 160 threonine) genotype of rs2243639 was protective against TB.

\section{Binding Activities of Four Variants of rSP-D to $M$. bovis BCG}

To confirm that SP-D 11Thr/160Thr (amino acid residues 11 and 160, both threonine) confer greater protection against TB infection as per the genetic analysis, as well as to further investigate the protective effects of four genetic variants of rSP-D in the progression of $\mathrm{TB}$ infection to $\mathrm{TB}$ disease, we constructed four genetic variants of SP-D, and then, respectively, measured

TABLE 1 | Demographic data of the study subjects.

\begin{tabular}{lccl}
\hline & Tuberculosis (TB) & Non-TB & \\
\hline $\begin{array}{l}\text { Age (years, } \\
\text { mean } \pm \text { SD) }\end{array}$ & $55.22 \pm 20.09$ & $57.75 \pm 11.08$ & $P=0.060(t=1.884)$ \\
Gender & & & \\
Male & 256 & 108 & $P<0.0001\left(\chi^{2}=30.600\right)$ \\
Female & 81 & 96 &
\end{tabular}

their protective ability against $M$. bovis BCG in vitro (SP-D binds to the surface of pathogens as a first line of defense to prevent pathogens binding before TB infection). We analyzed the binding activities of the four genetic variants of rSP-D with $M$. bovis BCG and found that all four genetic variants of rSP-D bound to $M$. bovis BCG in a dose-dependent and specific manner that could be inhibited by $10 \mathrm{mM}$ EDTA or $100 \mathrm{mM}$ maltose, but not by $100 \mathrm{mM}$ glucose (Figures $\mathbf{3 A}, \mathbf{B}$ ). At particular concentrations $(1 \mu \mathrm{g} / \mathrm{ml})$ of these four SP-D mutants, a change in the amino acid at residue 11 [from Met to Thr, i.e., rSP-D (92C/538A) and rSP-D (92C/538G)] led to higher binding activities with $M$. bovis BCG when compared with the other variants of rSP-D [rSP-D (92T/538A) and rSP-D (92T/538G)] (Figure 3B). Double reciprocal plot analyses revealed that the dissociation constant of rSP-D (92C/538A) binding to $M$. bovis was approximately sevenfold less than that for rSP-D (92T/538A) $\left[4.5 \times 10^{9} \mathrm{M}\right.$ for $\mathrm{rSP}-\mathrm{D}$ $(\mathrm{C} / \mathrm{A}), 3.1 \times 10^{8} \mathrm{M}$ for $\left.\mathrm{rSP}-\mathrm{D}(92 \mathrm{~T} / 538 \mathrm{~A})\right]$, indicating that the avidity of SP-D binding to $M$. bovis is affected by the change in amino acid at residue 11. By contrast, the change in amino acid residue 160 (from Thr to Ala) did not lead to a significant difference in binding ability to $M$. bovis BCG.

\section{Effects of the Four rSP-D Variants on Phagocytosis of MH-S Cells}

All rSP-D variants inhibited phagocytosis by alveolar macro_ phages following $M$. bovis BCG infection, but with different potencies. As the results presented in Figure 4A show, the change in amino acid at residue 11 (from Met to $\mathrm{Thr}$, i.e., 92T $\rightarrow$ 92C) led to significant inhibition of phagocytosis. The SP-D variant $11 \mathrm{M} / 160 \mathrm{~A}(92 \mathrm{~T} / 538 \mathrm{G})$ showed a weaker inhibition effect than the other variants (Figure 4A). The inhibitory effect of rSP-D could be attenuated by adding maltose as a competitor for bacterial binding.

\section{Determination of Aggregation and Chemotaxis of Infected MH-S Cells to Nearby Responsive Cells}

Aggregation of infected MH-S cells, an event in which a collection of macrophages is formed during mycobacterial infection, was also evaluated. The rSP-D variant 11Thr/160 Thr (92C/538A) inhibited the aggregation of infected $\mathrm{MH}-\mathrm{S}$ cells more obviously than the other variants (Figure 4B). The protein with $\mathrm{Thr}$ at residue 11 [rSP-D (92C/538A) and (92C/538G)] showed a significantly

TABLE 2 | Genotyping results and odds ratio analysis of the associations between surfactant proteins D single-nucleotide polymorphisms (SNPs) and tuberculosis (TB).

\begin{tabular}{|c|c|c|c|c|c|c|}
\hline SNP/genotype & TB & Non-TB & $\boldsymbol{P}$ & Adj. $P$ & Adj. OR ( $95 \%$ confidence interval) & $\boldsymbol{P}$ \\
\hline \multicolumn{7}{|l|}{ rs721917 (C92T) } \\
\hline CC (ref.) & $134(36.8 \%)$ & $80(45.2 \%)$ & 0.050 & 0.104 & & \\
\hline CT & $176(48.6 \%)$ & $82(46.3 \%)$ & $\chi^{2}=5.996$ & $\chi^{2}=4.519$ & $1.27(0.85,1.86)$ & 0.240 \\
\hline Tा & $54(14.8 \%)$ & 15 (9\%) & & & $1.95(1.01,3.75)$ & 0.045 \\
\hline \multicolumn{7}{|c|}{ rs2243639 (A538G) } \\
\hline GG (ref.) & $263(72.2 \%)$ & 129 (72.9\%) & 0.061 & 0.033 & & \\
\hline$A G$ & $96(26.4)$ & 40 (22.6\%) & $\chi^{2}=5.587$ & $\chi^{2}=6.853$ & $1.15(0.74,1.78)$ & 0.545 \\
\hline AA & $5(1.4 \%)$ & $8(1.4 \%)$ & & & $0.23(0.07,0.75)$ & 0.015 \\
\hline
\end{tabular}

Adj., adjusted by age and gender using logistic regression; ref., reference genotype. 


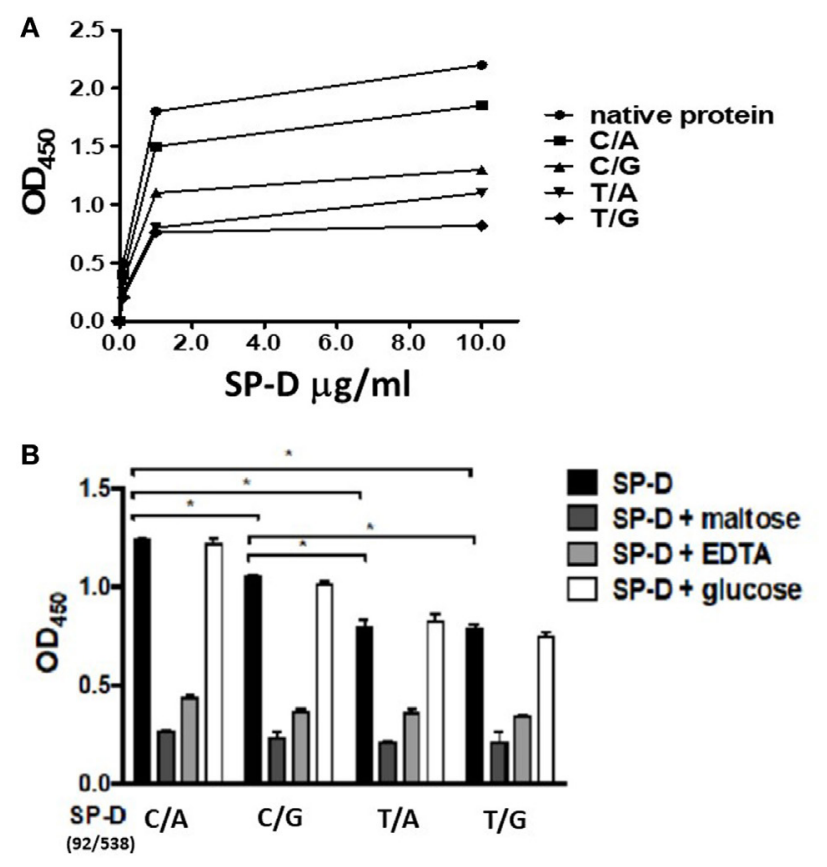

FIGURE 3 | (A) Dose-binding curves of native and four genetic variants of recombinant SP-D (rSP-D) proteins to Mycobacterium bovis bacillus Calmette-Guérin (M. bovis BCG). rSP-D protein (1 and $10 \mu \mathrm{g} / \mathrm{ml}$ ) were added to wells coated with ultraviolet-killed $M$. bovis BCG $\left(10^{6}\right.$ bacteria), then quantitated using solid-phase bacterial ELISA. (B) Inhibition effects of maltose and EDTA on the binding activity of four variants of rSP-D to M. bovis BCG. Surfactant proteins D (SP-D) protein $(1 \mu \mathrm{g} / \mathrm{ml})$ in PBS with $2 \mathrm{mM} \mathrm{CaCl}_{2}$ was added into M. bovis BCG-coated wells alone, or with $10 \mathrm{mM}$ EDTA, or $100 \mathrm{mM}$ maltose, or $100 \mathrm{mM}$ glucose. The binding activity to $M$. bovis BCG of rSP-D with residue 11Thr [(92C/538A) and (92C/538G)] was significantly higher than that of rSP-D with residue $11 \mathrm{Met}[(92 \mathrm{~T} / 538 \mathrm{~A})$ and (92T/538G)] ( $\left.{ }^{\star} P<0.05\right)$. C/A: 11Thr/160Thr; C/G: 11Thr/160Ala; T/A: 11Met/Thr; T/G: 11Met/160Ala.

stronger inhibitive effect than that with Met at residue 11 [rSP-D (92T/538A) and (92T/538G)]. The protein with $\mathrm{Thr}$ at residue 160 showed stronger inhibition than Ala 160 only when residue 11 was Thr and under the following conditions: $1 \mu \mathrm{g} / \mathrm{ml}$ SP-D or $1 \mu \mathrm{g} / \mathrm{ml}$ SP-D $+100 \mathrm{mM}$ glucose.

We further analyzed the associations between aggregation of $\mathrm{MH}-\mathrm{S}$ cells and various rSP-D mutants through a chemotaxis assay. However, the recruitment of nearby MH-S cells after mycobacterium infection did not differ between the four rSP-D variants (Figure 4C).

\section{The Associations Between the Four Variants of rSP-D and Viability of Infected MH-S Cells}

When immune cells first encounter a pathogen in the host, macrophages are one of the major cell types to confront the bacterial invasion, and the response of the cells is crucial, as it controls the outcome of infection. To further elucidate the effect of rSP-D on $\mathrm{MH}-\mathrm{S}$ cells infected by $M$. bovis BCG, a cell survival assay was performed using CCK-8. All variants of rSP-D improved the survival of infected $\mathrm{MH}-\mathrm{S}$ cells in a concentration-dependent

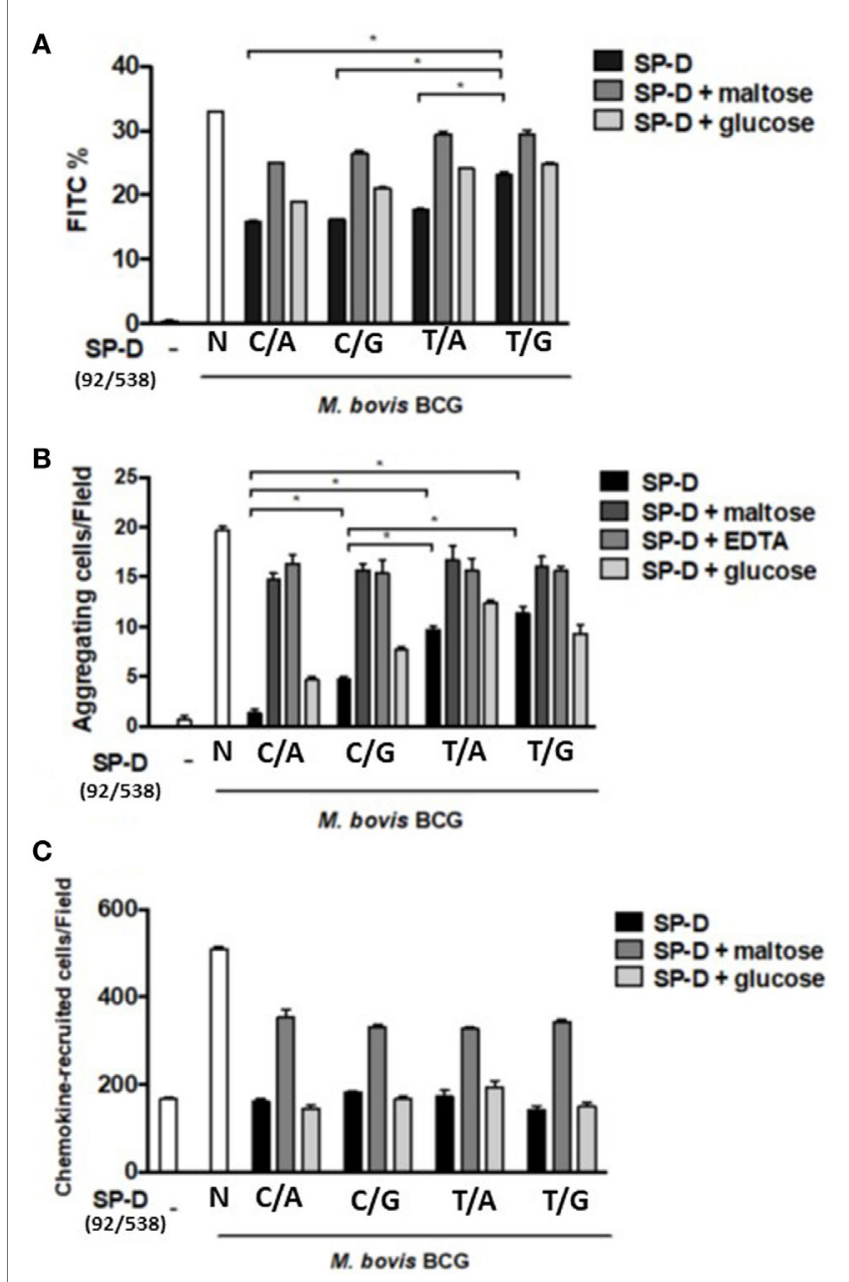

FIGURE 4 | (A) Inhibition of alveolar macrophage phagocytosis of Mycobacterium bovis bacillus Calmette-Guérin ( $M$. bovis BCG) by native and four genetic variants of recombinant SP-D (rSP-D) protein. Macrophages infected with FITC-labeled $M$. bovis BCG were analyzed using flow cytometry to assess the effects of the four rSP-D variants on the phagocytosis of $\mathrm{MH}-\mathrm{S}$ cells. rSP-D with residue 11Thr [(92C/538A) and (92C/538G)] inhibited phagocytosis of $M$. bovis BCG by $\mathrm{MH}-\mathrm{S}$ cells to a significantly greater extent than rSP-D with residue 11Met [(92T/538A) and (92T/538G)] $\left({ }^{\star} P<0.05\right)$. (B) Inhibition of agglutination of $M$. bovis BCG-infected $\mathrm{MH}$-S cells by native and four genetic variants of rSP-D proteins. The method of assessment of agglutination of $\mathrm{MH}-\mathrm{S}$ cells after mycobacterial infection is described in Section "Materials and Methods." rSP-D with residue 11Thr [(92C/538A) and (92C/538G)] inhibited the aggregation of $\mathrm{MH}-\mathrm{S}$ cells to a significantly greater extent than rSP-D with residue 11Met [(92T/538A) and (92T/538G)] ( $\left.{ }^{\star} P<0.05\right)$. (C) Inhibition of cell migration of $M$. bovis BCG-infected MH-S cells by native and four genetic variants of rSP-D proteins. All four rSP-D variants inhibited cell migration of infected $\mathrm{MH}-\mathrm{S}$ cells to nearby responsive cells in a concentration-dependent manner, but no significant difference was found between the four variants of rSP-D proteins. C/A: 11Thr/160Thr; C/G: 11Thr/160Ala; T/A: 11Met/Thr; T/G: 11Met/160Ala.

manner (Figure 5A). $1 \mu \mathrm{g} / \mathrm{ml}$ rSP-D with residue $11 \mathrm{Thr}$ $[(92 \mathrm{C} / 538 \mathrm{~A})$ and $(92 \mathrm{C} / 538 \mathrm{G})]$ demonstrated the most significant protection of infected $\mathrm{MH}-\mathrm{S}$ cells from cell death. In the rSP-D variants supplemented with $100 \mathrm{mM}$ maltose, a protective effect was not found, and no change to cell survival was observed with treatment supplemented with $100 \mathrm{mM}$ glucose. 


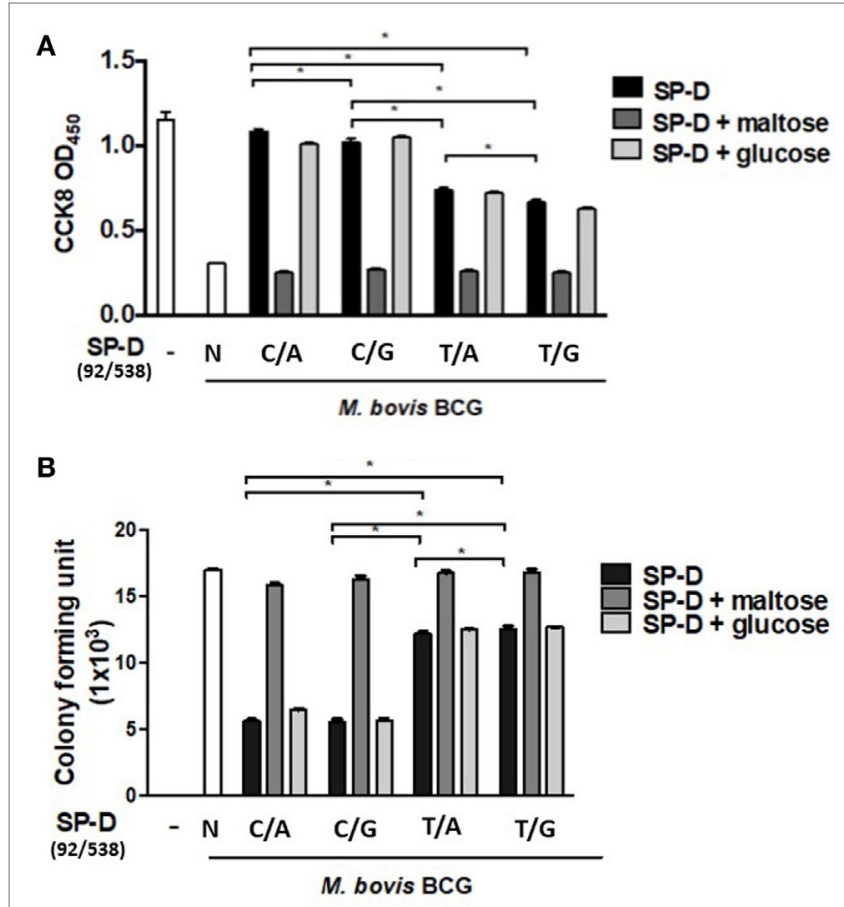

FIGURE 5 | (A) Cell survival of Mycobacterium bovis bacillus CalmetteGuérin ( $M$. bovis BCG)-infected $\mathrm{MH}-\mathrm{S}$ cells by native and four genetic variant recombinant SP-D (rSP-D) proteins measured using a Cell Counting Kit-8 assay. rSP-D with residue 11Thr [(92C/538A) and (92C/538G)] exhibited significantly better survival of infected $\mathrm{MH}$-S cells than $\mathrm{rSP}-\mathrm{D}$ with residue 11 Met $[(92 \mathrm{~T} / 538 \mathrm{~A})$ and $(92 \mathrm{~T} / 538 \mathrm{G})]\left({ }^{\star} P<0.05\right)$. (B) Intracellular variability of $M$. bovis BCG after phagocytosis by alveolar macrophages treated with native and four genetic variants of rSP-D proteins. rSP-D with residue 11Thr [(92C/538A) and (92C/538G)] inhibited $M$. bovis BCG intracellular growth significantly more than rSP-D with residue 11Met [(92T/538A) and (92T/538G)] ( $\left.{ }^{\star} P<0.05\right)$. TT: 11T/160T; TA: 11T/160A; MT: 11M/160A; MA: 11M/160A.

\section{The Effects of rSP-D Variants on Intracellular $M$. bovis BCG Growth}

The TB survival rate within macrophages is a hallmark of persistent (TB) pathogenesis. Aliquots of infected MH-S cell lysate were plated onto $7 \mathrm{H} 11$ agar to evaluate the effects of the four rSP-D variants on intracellular bacteria growth. Our results showed that all four variants of rSP-D inhibited intracellular M. bovis BCG growth (Figure 5B). The addition of $100 \mathrm{mM}$ maltose reversed the inhibition, while no effect was observed with the addition of $100 \mathrm{mM}$ glucose. Compared with residue $11 \mathrm{Met}$ [(92T/538A) and $(92 \mathrm{~T} / 538 \mathrm{G})]$, rSP-D variants with residue 11 Thr $[(92 \mathrm{C} / 538 \mathrm{~A})$ and $(92 \mathrm{C} / 538 \mathrm{G})]$ significantly inhibited the intracellular growth of $M$. bovis BCG (Figure 5B). There was no difference in growth whether residue 160 was bearing Ala or Thr.

\section{DISCUSSION}

In this study, we used M. bovis BCG, a good surrogate representing $M T B$, to study the role of SP-D polymorphism in mycobacterial infection and the connection with TB. Our results showed that $M$. bovis BCG interacted with the different rSP-D variants at various levels; these effects were abolished following the addition of maltose or EDTA, which causes a competitive effect with the lectin domain of SP-D or depletion of calcium. At first glance, it appeared that interaction of SP-D with MTB was dependent on its carbohydrate recognition domain (CRD). However, a closer look at the comparison of innate immunity against TB infection of genetic variants in the N-terminal and collagenous regions of rSP-D showed different degrees of activity. We found that SP-D residue 11 polymorphism, Thr (92C) and Met (92T), affected the binding activity of SP-D to M. bovis BCG, phagocytosis of infected MH-S cells, aggregation of infected $\mathrm{MH}-\mathrm{S}$ cells, cell death of infected MH-S cells, and intracellular growth of $M$. bovis BCG. By contrast, the effects of SP-D 160 polymorphism, Ala (538G) and Thr (538A), were weaker than those of residue 11 polymorphism, and did not appear distinctly. From the results of SP-D variant functional and genetic analysis, residue $11 \mathrm{Met}$ (92T) appeared likely to cause susceptibility to TB, which was validated in our TB-infected patients.

The SP-D gene contains a total of eight exons, seven of which are coding. Coding and non-coding SNPs (rs721917, rs6413520, rs2243639, rs3088308, rs1051246, rs1923537, rs2245121, rs911887, rs2255601, and rs7078012) are also found within the SP-D gene $(31,32)$; most of these have been found to be associated with a number of diseases $(23,33,34)$. Among the known SP-D SNPs, two major polymorphisms, C92T (rs721917) and A538G (rs2243639), were chosen for analysis in this study. The rs721917 SFTPD SNP, known as C92T and SP-D A11, located on exon 1 and a short non-collagen-like $\mathrm{N}$-terminal section, is associated with serum levels of SP-D (35), and explains $39 \%$ of the phenotypic variation (36), the Met11 (92T) allelic variant being associated at the highest level (37). Consistent with genetic determination of SP-D levels, the constitutive distribution of multimerized (high molecular weight, HMW) and non-multimerized (low molecular weight, LMW) SP-D in human body fluids is also genetically determined, with individuals homozygous for the Met11 allele having a relative predominance of HMW SP-D, and Thr11 (92C) allele homozygotes having more LMW SP-D (37). This change in the multimerization of SP-D may also affect its biological functions in vitro and in vivo (36). In fact, several studies have linked rs721917 with diseases (38), indicating involvement of SP-D level and/or size variation in disease pathogenesis. Study has shown that amino acid position 343 of SP-D has a critical role in the selective binding of glycolipids from MTB (39); however, no polymorphism exists in this genetic position.

Another coding single-nucleotide variation is located on exon 4 , and the collagen-like region in the mature protein is rs2243639, known as A538G and SPD A160. As with other genetic alleles, the two-marker SP-D/SP-A haplotype, DA160_A/SP-A2 1A, is protective against the development of neonatal respiratory distress syndrome (40). SP-A2/SP-D haplotypes were also found to protect against bronchopulmonary dysplasia: 1A2-Ala160 (rs2243639) and 1A2-Thr11 (rs721917)-Ala160 (rs2243639) (41). It has been suggested that the protective effect of SP-D Ala160 in bronchopulmonary dysplasia is likely due to interactions with other gene(s). In our study of the interaction with $M$. bovis BCG, residue 160 Ala (92C/538G) showed a lesser binding 
activity than $160 \mathrm{Thr}(92 \mathrm{C} / 538 \mathrm{~A})$ when residue 11 was Thr, but not when it was Met. The SP-D variant 11M/160A (92T/538G) showed weaker inhibition of phagocytosis than the other variants at an SP-D concentration of $1 \mu \mathrm{g} / \mathrm{ml}$. Our results implied that polymorphism A539G (160 residue Ala/Thr) has less impact than C92T (11 residue Thr/Met) and may exhibit an effect only under specific conditions or through interaction with other genetic factors. The protective effect of homozygous 538A (160 residue Thr) against $\mathrm{TB}$ was consistent with the results of functional studies mentioned above.

Surfactant proteins D binds to the bacterial surface and reduces the uptake of bacteria by alveolar macrophages (19). Also, SP-D polymorphisms have been demonstrated to be associated with the risk of TB $(23,42)$. A genetic study of a Mexican population identified that SP-D A11C (amino acid residue 11, risk allele threonine) was associated with subgroups of patients with TB by comparing a TB group and a tuberculin-skin test-positive group (symptom-free and normal chest radiographs). The association was not found when the TB group was compared with a control group (23). Our association study revealed that homozygous residue 11 Met was a risk genotype for TB, but this is not in agreement with the conclusions of the above study.

In this study, we demonstrated that the genetic variants of rSP-D in the $\mathrm{N}$ terminal and collagenous region confer different degrees of innate immunity against $\mathrm{TB}$ infection in vitro. Therefore, it is likely that SP-D polymorphisms C92T and A538G affect susceptibility to TB, which is in agreement with our findings in an association study of clinical TB patients. There were some limitations to our study. First, we were not able to differentiate whether or not these genetic variants of rSP-D undergo multimerization (HMW vs LMW) under our experimental conditions. HMW SP-D multimers are only partly dependent on disulfide crosslinking of the $\mathrm{N}$ terminal region, and some SP-D subunits are non-covalently associated depending on the protein concentration in the solution (36). Several studies have shown some discrepancies in binding characteristics and activities between HMW and LMW SP-D $(36,37,43)$. Second, we were not able to demonstrate in vivo effects of the genetic variants of rSP-D in a TB-infected animal model due to a small yield of recombinant full-length human SP-D from HEK 293 cells. Third, although high antigenic and structural similarities exist between $M T B$ and $M$. bovis BCG, use of $M$. bovis might not wholly represent the infectious nature of $M T B$ in hosts. MTB could not be utilized in this study due to a lack of availability of the necessary safety equipment in our laboratory.

However, to evaluate the influences of SP-D polymorphisms in vivo and in lung diseases, wild-type and transgenic Sftpd $d^{-1-}$ mice

\section{REFERENCES}

1. Maher D, Raviglione M. Global epidemiology of tuberculosis. Clin Chest Med (2005) 26:167-82. doi:10.1016/j.ccm.2005.02.009

2. WHO. Multidrug and Extensively Drug-Resistant TB (M/XDRTB):2010 Global Report on Surveillance and Response. Geneva, Switzerland: World Health Organization (2010).

3. WHO. Global Tuberculosis Report 2014. Geneva, Switzerland: World Health Organization (2014).

4. CDCs Taiwan. Taiwan Tuberculosis Control Report. Taiwan, Republic of China: Centers for Disease Control, Department of Health (2012). expressing either the human SP-D Met11 or Thr11 allelic variants were generated (44-46). In an acute model of allergic asthma, transgenic mice were sensitized and challenged with ovalbumin, and the results showed that murine expression of human polymorphic SP-D variants in the $\mathrm{N}$-terminal region does not significantly influence the severity of allergic airway inflammation (46). Other studies also showed that low transgene expression levels and differences in the distribution of human rSP-D in the lung made comparison of phenotypes between wild-type and transgenic mice difficult $(44,45)$. In fact, a $60-\mathrm{kDa}$ recombinant trimeric fragment of SP-D, without the $\mathrm{N}$-terminus but maintaining part of the collagen region, appeared to have comparable effects to native SP-D in in vivo and in vitro studies, which implies that the homeostatic and anti-microbial effects of SP-D are primarily mediated by its CRD region $(47,48)$.

In conclusion, our results demonstrated the role of SP-D in innate immunity against TB infection. Although this mostly depends on the binding of CRD with $M T B$, this study elucidates that it is also influenced by the genetic variants in the $\mathrm{N}$-terminal and collagenous domains of SP-D. The two major SP-D polymorphisms C92T and A538G, and especially C92T, appeared to affect susceptibility to TB. Our association study of the susceptibility to TB infection in patients also supported this hypothesis.

\section{ETHICS STATEMENT}

The study protocol conformed to the ethical guidelines of the 1975 Declaration of Helsinki and was approved by the Ethics Committees of Taoyuan General Hospital and National Cheng Kung University Hospital.

\section{AUTHOR CONTRIBUTIONS}

$\mathrm{M}-\mathrm{HH}, \mathrm{C}-\mathrm{YO}, \mathrm{W}-\mathrm{YH}, \mathrm{J}-\mathrm{YW}$, and LW contributed to the conception and design of the study; $\mathrm{M}-\mathrm{HH}$ and $\mathrm{W}-\mathrm{YH}$ performed experiments; $\mathrm{C}-\mathrm{YO}$ and $\mathrm{H}-\mathrm{FK}$ analyzed the data; $\mathrm{C}-\mathrm{YO}$ and S-WL collected TB patients and performed genetic analysis; LW and J-YW wrote the first draft of the manuscript; and all authors contributed to manuscript revision, and read and approved the submitted version.

\section{FUNDING}

This project was supported by Ministry of Science and Technology (MOST) and Academia Sinica (AS) grants: MOST 107-2321-B-006-008; MOST 104-2321-B-006-008; MOST 1032321-B-006-030; AS-106-TP-B09.

5. Comstock GW. Tuberculosis in twins: a re-analysis of the Prophit survey. Am Rev Respir Dis (1978) 117:621-4.

6. van der Eijk EA, van de Vosse E, Vandenbroucke JP, van Dissel JT. Heredity versus environment in tuberculosis in twins: the 1950s United Kingdom Prophit Survey Simonds and Comstock revisited. Am J Respir Crit Care Med (2007) 176:1281-8. doi:10.1164/rccm.200703-435OC

7. Bellamy R, Beyers N, McAdam KP, Ruwende C, Gie R, Samaai P, et al. Genetic susceptibility to tuberculosis in Africans: a genome-wide scan. Proc Natl Acad Sci U S A (2000) 97:8005-9. doi:10.1073/pnas.140201897

8. Cooke GS, Campbell SJ, Bennett S, Lienhardt C, McAdam KP, Sirugo G, et al. Mapping of a novel susceptibility locus suggests a role for MC3R and 
CTSZ in human tuberculosis. Am J Respir Crit Care Med (2008) 178:203-7. doi:10.1164/rccm.200710-1554OC

9. Mahasirimongkol S, Yanai H, Nishida N, Ridruechai C, Matsushita I, Ohashi J, et al. Genome-wide SNP-based linkage analysis of tuberculosis in Thais. Genes Immun (2009) 10:77-83. doi:10.1038/gene.2008.81

10. Miller EN, Jamieson SE, Joberty C, Fakiola M, Hudson D, Peacock CS, et al. Genome-wide scans for leprosy and tuberculosis susceptibility genes in Brazilians. Genes Immun (2004) 5:63-7. doi:10.1038/sj.gene.6364031

11. Farhat MR, Shapiro BJ, Kieser KJ, Sultana R, Jacobson KR, Victor TC, et al. Genomic analysis identifies targets of convergent positive selection in drugresistant Mycobacterium tuberculosis. Nat Genet (2013) 45:1183-9. doi:10.1038/ng.2747

12. Mahasirimongkol S, Yanai H, Mushiroda T, Promphittayarat W, Wattanapokayakit S, Phromjai J, et al. Genome-wide association studies of tuberculosis in Asians identify distinct at-risk locus for young tuberculosis. J Hum Genet (2012) 57:363-7. doi:10.1038/jhg.2012.35

13. Thye T, Vannberg FO, Wong SH, Owusu-Dabo E, Osei I, Gyapong J, et al. Genome-wide association analyses identifies a susceptibility locus for tuberculosis on chromosome 18q11.2. Nat Genet (2010) 42:739-41. doi:10.1038/ ng.639

14. Moller M, Hoal EG. Current findings, challenges and novel approaches in human genetic susceptibility to tuberculosis. Tuberculosis (Edinb) (2010) 90:71-83. doi:10.1016/j.tube.2010.02.002

15. Azad AK, Sadee W, Schlesinger LS. Innate immune gene polymorphisms in tuberculosis. Infect Immun (2012) 80:3343-59. doi:10.1128/IAI.00443-12

16. Wang JY, Reid KB. The immunoregulatory roles of lung surfactant collectins SP-A, and SP-D, in allergen-induced airway inflammation. Immunobiology (2007) 212:417-25. doi:10.1016/j.imbio.2007.01.002

17. Torrelles JB, Azad AK, Henning LN, Carlson TK, Schlesinger LS. Role of C-type lectins in mycobacterial infections. Curr Drug Targets (2008) 9:102-12. doi:10.2174/138945008783502467

18. Gaynor CD, McCormack FX, Voelker DR, McGowan SE, Schlesinger LS. Pulmonary surfactant protein A mediates enhanced phagocytosis of Mycobacterium tuberculosis by a direct interaction with human macrophages. J Immunol (1995) 155:5343-51.

19. Ferguson JS, Voelker DR, McCormack FX, Schlesinger LS. Surfactant protein D binds to Mycobacterium tuberculosis bacilli and lipoarabinomannan via carbohydrate-lectin interactions resulting in reduced phagocytosis of the bacteria by macrophages. J Immunol (1999) 163:312-21.

20. Ferguson JS, Voelker DR, Ufnar JA, Dawson AJ, Schlesinger LS. Surfactant protein D inhibition of human macrophage uptake of Mycobacterium tuberculosis is independent of bacterial agglutination. J Immunol (2002) 168:1309-14. doi:10.4049/jimmunol.168.3.1309

21. Ferguson JS, Martin JL, Azad AK, McCarthy TR, Kang PB, Voelker DR, et al. Surfactant protein D increases fusion of Mycobacterium tuberculosiscontaining phagosomes with lysosomes in human macrophages. Infect Immun (2006) 74:7005-9. doi:10.1128/IAI.01402-06

22. Haataja R, Hallman M. Surfactant proteins as genetic determinants of multifactorial pulmonary diseases. Ann Med (2002) 34:324-33. doi:10.1080/ 078538902320772089

23. Floros J, Lin HM, Garcia A, Salazar MA, Guo X, DiAngelo S, et al. Surfactant protein genetic marker alleles identify a subgroup of tuberculosis in a Mexican population. J Infect Dis (2000) 182:1473-8. doi:10.1086/315866

24. Madan T, Saxena S, Murthy KJ, Muralidhar K, Sarma PU. Association of polymorphisms in the collagen region of human SP-A1 and SP-A2 genes with pulmonary tuberculosis in Indian population. Clin Chem Lab Med (2002) 40:1002-8. doi:10.1515/CCLM.2002.174

25. Malik S, Greenwood CM, Eguale T, Kifle A, Beyene J, Habte A, et al. Variants of the SFTPA1 and SFTPA2 genes and susceptibility to tuberculosis in Ethiopia. Hum Genet (2006) 118:752-9. doi:10.1007/s00439-005-0092-y

26. Yang HY, Li H, Wang YG, Xu CY, Zhao YL, Ma XG, et al. Correlation analysis between single nucleotide polymorphisms of pulmonary surfactant protein A gene and pulmonary tuberculosis in the Han population in China. Int J Infect Dis (2014) 26:31-6. doi:10.1016/j.ijid.2014. 03.1395

27. Sorensen GL, Husby S, Holmskov U. Surfactant protein A and surfactant protein D variation in pulmonary disease. Immunobiology (2007) 212: 381-416. doi:10.1016/j.imbio.2007.01.003
28. Lahti M, Lofgren J, Marttila R, Renko M, Klaavuniemi T, Haataja R, et al. Surfactant protein D gene polymorphism associated with severe respiratory syncytial virus infection. Pediatr Res (2002) 51:696-9. doi:10.1203/ 00006450-200206000-00006

29. Ou CY, Chen CZ, Hsiue TR, Lin SH, Wang JY. Genetic variants of pulmonary SP-D predict disease outcome of COPD in a Chinese population. Respirology (2015) 20:296-303. doi:10.1111/resp.12427

30. Venisse A, Berjeaud JM, Chaurand P, Gilleron M, Puzo G. Structural features of lipoarabinomannan from Mycobacterium bovis BCG. Determination of molecular mass by laser desorption mass spectrometry. J Biol Chem (1993) 268:12401-11.

31. Lu J, Willis AC, Reid KB. Purification, characterization and CDNA cloning of human lung surfactant protein D. Biochem J (1992) 284:795-802. doi:10.1042/ bj2840795

32. Crouch E, Persson A, Chang D, Heuser J. Molecular structure of pulmonary surfactant protein D (SP-D). J Biol Chem (1994) 269:17311-9.

33. Foreman MG, Kong X, DeMeo DL, Pillai SG, Hersh CP, Bakke P, et al. Polymorphisms in surfactant protein-D are associated with chronic obstructive pulmonary disease. Am J Respir Cell Mol Biol (2011) 44:316-22. doi:10.1165/rcmb.2009-0360OC

34. Hilgendorff A, Heidinger K, Bohnert A, Kleinsteiber A, Konig IR, Ziegler A, et al. Association of polymorphisms in the human surfactant protein-D (SFTPD) gene and postnatal pulmonary adaptation in the preterm infant. Acta Paediatr (2009) 98:112-7. doi:10.1111/j.1651-2227.2008. 01014.x

35. Heidinger K, Konig IR, Bohnert A, Kleinsteiber A, Hilgendorff A, Gortner L, et al. Polymorphisms in the human surfactant protein-D (SFTPD) gene: strong evidence that serum levels of surfactant protein-D (SP-D) are genetically influenced. Immunogenetics (2005) 57:1-7. doi:10.1007/s00251005-0775-5

36. Sorensen GL, Hoegh SV, Leth-Larsen R, Thomsen TH, Floridon C, Smith K, et al. Multimeric and trimeric subunit SP-D are interconvertible structures with distinct ligand interaction. Mol Immunol (2009) 46:3060-9. doi:10.1016/ j.molimm.2009.06.005

37. Leth-Larsen R, Garred P, Jensenius H, Meschi J, Hartshorn K, Madsen J, et al. A common polymorphism in the SFTPD gene influences assembly, function, and concentration of surfactant protein D. JImmunol (2005) 174:1532-8. doi:10.4049/jimmunol.174.3.1532

38. Sorensen GL. Surfactant protein D in respiratory and non-respiratory diseases. Front Med (2018) 5:18. doi:10.3389/fmed.2018.00018

39. Carlson TK, Torrelles JB, Smith K, Horlacher T, Castelli R, Seeberger PH, et al. Critical role of amino acid position 343 of surfactant protein-D in the selective binding of glycolipids from Mycobacterium tuberculosis. Glycobiology (2009) 19:1473-84. doi:10.1093/glycob/cwp122

40. Thomas NJ, Fan R, Diangelo S, Hess JC, Floros J. Haplotypes of the surfactant protein genes A and D as susceptibility factors for the development of respiratory distress syndrome. Acta Paediatr (2007) 96:985-9. doi:10.1111/j.1651-2227.2007.00319.x

41. Pavlovic J, Papagaroufalis C, Xanthou M, Liu W, Fan R, Thomas NJ, et al. Genetic variants of surfactant proteins A, B, C, and D in bronchopulmonary dysplasia. Dis Markers (2006) 22:277-91. doi:10.1155/2006/817805

42. Vaid M, Kaur S, Taruna M, Singh H, Gupta V, Murthy K, et al. Association of SP-D, MBL and I-NOS genetic variants with pulmonary tuberculosis. Indian J Human Genet (2006) 12:105-10. doi:10.4103/09716866.29851

43. Fakih D, Akiki Z, Junker K, Medlej-Hashim M, Waked M, Salameh P, et al. Surfactant protein D (SP-D) levels, polymorphisms and multimerization in COPD and asthma. Respirology (2018) 23(3):298-305. doi:10.1111/ resp. 13193

44. Knudsen L, Ochs K, Boxler L, Tornoe I, Lykke-Sorensen G, Mackay RM, et al. Surfactant protein D (SP-D) deficiency is attenuated in humanised mice expressing the Met(11)Thr short nucleotide polymorphism of SP-D: implications for surfactant metabolism in the lung. J Anat (2013) 223:581-92. doi:10.1111/joa.12120

45. Kingma PS, Zhang L, Ikegami M, Hartshorn K, McCormack FX, Whitsett JA. Correction of pulmonary abnormalities in $\mathrm{Sftpd}^{-/-}$mice requires the collagenous domain of surfactant protein D. J Biol Chem (2006) 281:24496-505. doi:10.1074/jbc.M600651200 
46. Winkler C, Bahlmann O, Viereck J, Knudsen L, Wedekind D, Hoymann HG, et al. Impact of a Met(11)Thr single nucleotide polymorphism of surfactant protein D on allergic airway inflammation in a murine asthma model. Exp Lung Res (2014) 40:154-63. doi:10.3109/01902148.2014.891062

47. Kishore U, Wang JY, Hoppe HJ, Reid KB. The alpha-helical neck region of human lung surfactant protein D is essential for the binding of the carbohydrate recognition domains to lipopolysaccharides and phospholipids. Biochem J (1996) 318:505-11. doi:10.1042/bj3180505

48. Madan T. Recombinant fragment of human surfactant protein D: a hierarchical regulator of pulmonary hypersensitivity. Am J Respir Crit Care Med (2017) 196:1495-6. doi:10.1164/rccm.201709-1934ED
Conflict of Interest Statement: The authors declare that the research was conducted in the absence of any commercial or financial relationships that could be construed as a potential conflict of interest.

Copyright (C) 2018 Hsieh, Ou, Hsieh, Kao, Lee, Wang and Wu. This is an open-access article distributed under the terms of the Creative Commons Attribution License (CC BY). The use, distribution or reproduction in other forums is permitted, provided the original author(s) and the copyright owner(s) are credited and that the original publication in this journal is cited, in accordance with accepted academic practice. No use, distribution or reproduction is permitted which does not comply with these terms. 